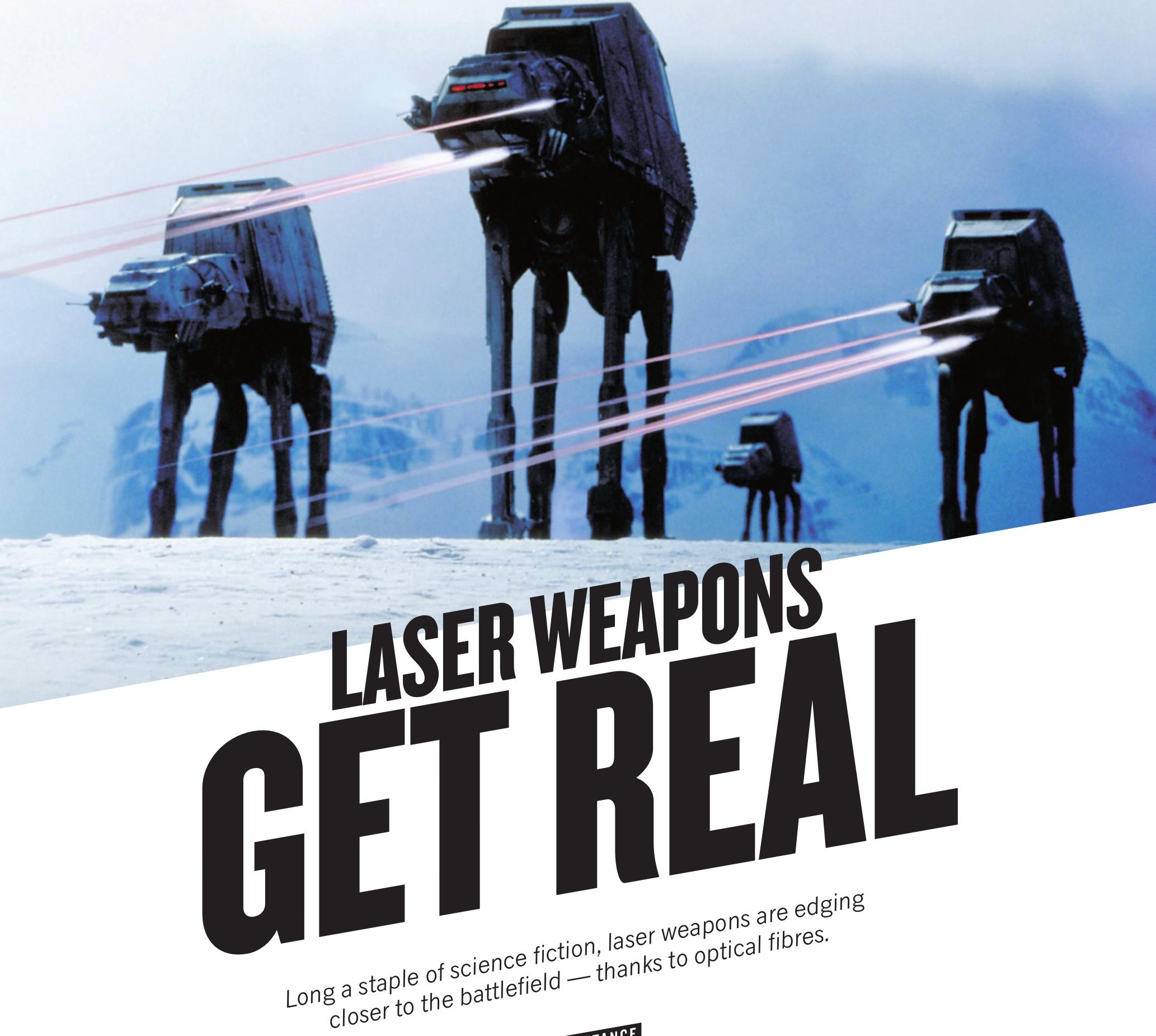

BY ANDY EXTANCE

S ilently, the drone aircraft glides above the arid terrain of New Mexico - until it suddenly pivots out of control and plummets to the ground.

Then a mortar round rises from its launcher, arcs high and begins to descend towards its target - only to flare and explode in mid-flight.

On the desert floor, on top of a big, sand-coloured truck, a cubic mechanism pivots and fires an invisible infrared beam to zap one target after another. This High Energy Laser Mobile Demonstrator (HEL MD) is a prototype laser weapon developed for the US Army by aerospace giant Boeing of Chicago, Illinois. Inside the truck, Boeing electrophysics engineer Stephanie Blount stares at the targets on her laptop's screen and directs the laser using a handheld game controller. "It has a very game-like feel," she says.

That seems only natural: laser weapons are a staple of modern video games, and ray-guns of various sorts were common in science fiction for decades before the first real-life laser was demonstrated in 1960 . But they are not a fantasy anymore. The Boeing prototype is just one of several such weapons developed in recent years in both the United States and Europe, largely thanks to the advent of relatively cheap, portable and robust lasers that generate their beams using optical fibres.

The output of these fibre weapons is measured in kilowatts $(\mathrm{kW})$, orders of magnitude less than the megawatt-class devices once envisioned for the US Strategic Defense Initiative - an ultimately unsuccessful cold-war plan that sought to use lasers to disable ballistic missiles carrying nuclear warheads.

But the modern, less ambitious, weapons are on the brink of realworld deployment. Tests such as those of the Boeing system show that the lasers have enough power to overcome threats from terror 


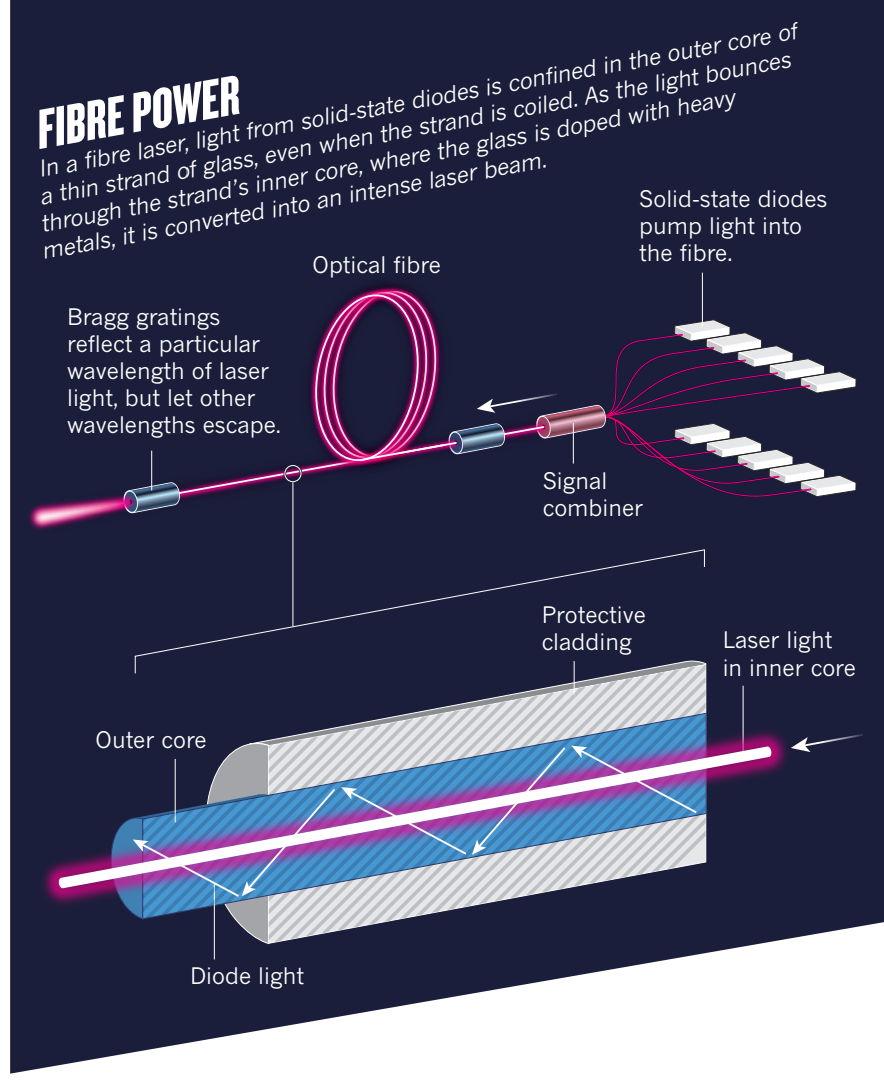

groups - at a fraction of the price of conventional defences. "It's a very cost-effective solution to taking out cheaply made weapons like small mortars or rockets made out of sewer pipe," says Blount.

In late 2014, for example, the US Navy showed that a ship-mounted laser-weapon system called LaWS could target small boats, such as those used by terrorists and pirates. That experimental weapon is currently installed on the USS Ponce, an amphibious support ship in the Gulf.

Many challenges to full-scale deployment remain, warn developers, from the need to boost the weapons' power to the difficulty of operating a laser in fog and clouds. But specialists in defence and security are starting to take lasers seriously. "After a nearly half-century quest, the US military today is on the cusp of finally fielding operationally relevant directed-energy weapons," wrote Paul Scharre, an advanced-technology specialist at the Washington DC-based Center for a New American Security (CNAS), in report on laser weapons released in April ${ }^{1}$.

\section{THE POWER PREDICAMENT}

Laser weapons have long fascinated weapons developers - most notably during the heyday of the Strategic Defense Initiative, nicknamed Star Wars, in the 1980s and 1990s. US spending on laser-weapons research peaked in 1989 when, according to the CNAS report ${ }^{1}$, the government spent the equivalent of US $\$ 2.4$ billion in 2014 dollars. Funding has continued at lower levels ever since. Yet the original goal, of being able to shoot down incoming ballistic missiles, proved unattainable.

The trick with any laser weapon is to focus its energy into a spot that is small enough to heat up and damage the target - and to do that with a machine that is compact and portable enough for the battlefield. This is easier said than done. In 1996, for example, the US Air Force initiated the Airborne Laser project as one of its contributions to defence against ballistic missiles. Because it was impossible at the time to generate the required megawatts of optical power electrically, the developers chose a chemical oxygen iodine laser (COIL) that could be fuelled by a chemical reaction. But the COIL was so bulky that it could only be carried on a Boeing 747 , and left little space for laser fuel. "It needed remote mixing units and chemicals weighing tens of thousands of pounds," says Paul Shattuck, head

\section{$\rightarrow$ NATURE.COM}

For a podcast on laser weapons, visit: go.nature.com/2xdtw4 of directed-energy systems for Lockheed Martin Space Systems, which provided the project's beam-control technology.

Another major problem was the atmosphere, says Phillip Sprangle, senior scientist for directed-energy physics at the Naval Research Laboratory in Washington DC. Not only was the beam scattered by dust and natural turbulence, he says, but its passage caused 'thermal blooming. When the beam propagated at very high powers, Sprangle explains, "the atmosphere absorbed laser light, heating the air and causing the laser beam to spread out". That spreading, in turn, dissipated the laser's energy.

The good news for the Airborne Laser project was that this issue, at least, had a solution: adaptive optics technology similar to that used by astronomers to clarify their view of the stars (see Nature 517, 430-432; 2015). The technology uses mirrors to automatically distort the laser beam in a way that cancels the effects of the turbulence, with the same result as a pair of glasses correcting for aberrations in the eye. "As the laser beam passes through the atmosphere," says Shattuck, "it cleans up, and it's nice and tight when it gets to the target."

By 2010, the adaptive optics was good enough for the Airborne Laser to destroy a ballistic missile in flight. By then, however, logistical issues such as the size problem had led the Department of Defense to lose its enthusiasm for energy weapons in general. It cancelled the Airborne Laser programme outright by early 2012. At the same time, the department's spending on high-energy lasers in general was falling; it dropped from \$961 million in 2007 to \$344 million in 2014 .

\section{FIBRES IN THE SPOTLIGHT}

The money did not vanish entirely: attention was already shifting to fibre lasers as a way to deliver results more economically. Fibre lasers were invented in 1963, and since the 1990s they have been advanced almost entirely by IPG Photonics in Oxford, Massachusetts. Whereas other solid-state lasers use rigid rods, slabs or discs of crystal to generate the beam, and so have to be fairly large, fibre lasers use thin optical fibres that can be wrapped into compact coils (see 'Fibre power'). The fibres can collect their optical energy from brighter versions of the cheap laser diodes used in DVD players, and then amplify the light to higher power, with overall electrical-to-optical conversion efficiencies greater than $30 \%$. This is at least double the efficiency typical of other solid-state lasers, and close to that of chemical lasers such as COIL. And, being intrinsically long and thin, the fibres have a high surface area to volume ratio and can radiate away waste heat very quickly — an ability that helps to give the lasers a long working life and low maintenance requirements.

These advantages first attracted attention during the 1990s, when fibre lasers began to be used to beef up optical signals carrying Internet data through undersea cables. But since the early 2000s, IPG has focused on developing kilowatt-class industrial lasers for welding, drilling and cutting - devices that also attracted the attention of military researchers.

Around 2010, recalls Shattuck, he and his colleagues at Lockheed Martin heard from Israeli civilians targeted by rockets launched from the Gaza Strip. "The mayor of a village stood up and said, 'Please, give me some kind of defence," Shattuck says. This inspired Lockheed Martin to develop the Area Defense Anti-Munitions (ADAM) system, which uses an off-the-shelf 10-kW laser from IPG to keep costs down. Since 2012, the company has shown that ADAM can disable targets such as boats, drones and simulated small-calibre rockets from about 1.5 kilometres away. Although unwilling to disclose the price of ADAM - or whether anybody has bought one - Lockheed Martin says that it is now ready to provide the system to customers.

Blount is less reticent about Boeing's HEL MD prototype, which also uses a commercial 10-kW fibre laser. With the system drawing its power from the vehicle engine or a separate generator, she says, "it takes less than two cups of fuel to fire the laser for long enough to disable many targets." This makes it much cheaper to use for defence than conventional missiles. "An inexpensive missile is $\$ 100,000$ and that's one shot," says David DeYoung, Boeing's director of directed-energy systems. "To shoot a laser-weapon system once is less than $\$ 10$."

Blount stresses that the resurgence of laser weapons owes at least as 
much to advanced image-recognition and targeting systems as to the laser itself. "The better the pointing and tracking system," she says, "the better able you are to put the beam on the most vulnerable point of a target."

Thanks to computerized aiming, HEL MD can operate in wholly autonomous mode, which Boeing tested successfully in May 2014 although the trials uncovered an unexpected challenge. The weapon's laser beam is silent and invisible, and not all targets explode as they are destroyed, so an automated battle can be over before operators have noticed anything. "The engagements happen quickly, and unless you're staring at a screen 24-7 you'll never see them," Blount says. "So we've built sound in for whenever we fire the laser. We plan on taking advantage of lots of Star Trek and Star Wars sound bites."

\section{STRENGTH IN NUMBERS}

Aiming and targeting may be battle-ready, but power is still a problem. A commercial laser's 10-kW output is at the low end of what is useful for laser weapons. And using fibres puts limits on the beam's power and quality - not least because at high powers, the cascade of photons surging through the fibre can heat it up faster than it can radiate the energy, and can thus cause damage. To avoid this, researchers are working to combine the output from several lasers.

The ideal way to do this would be 'coherent combining', in which the waves from each laser march together in tightly synchronized formation. This technique is widely used in radio and microwave applications, says Tso Yee Fan, a laser scientist at the Massachusetts Institute of Technology's defence-oriented Lincoln Laboratory in Lexington. But coherence is much tougher to achieve with visible and infrared light. The waves from each laser must have almost identical wavelengths, the planes of their oscillations must precisely align, and the peaks and troughs of each wave must coincide. "In radio-frequency or microwaves, the wavelength's a few centimetres," Fan says. "In optics, the wavelength's around a micrometre, so being able to do those kinds of controls has been really difficult."

But that may not matter much, says Sprangle. In 2006, he and his team reported computer simulations suggesting that an incoherent combination' of several fibre-laser beams hitting a single spot would be almost as effective as a coherent combination ${ }^{2}$. With either approach, he says, "when you're propagating over long ranges through atmospheric turbulence, you get approximately the same power on the target". In 2009, his group confirmed this theory by using mirrors to combine 4 fibre-laser beams into a 5-centimetre spot on a target more than 3 kilometres away ${ }^{3}$.

Building on Sprangle's work, the US Office of Naval Research has developed the 30-kW LaWS, which incoherently combines six commercial fibre-lasers. LaWS has been installed on the USS Ponce since September 2014, and has been tested on objects such as small boats and drones.

The missile-specialist MBDA Germany in Schrobenhausen has developed a similar approach ${ }^{4}$. In October 2012, the firm successfully used its 40-kW combined fibre-beam system to destroy model artillery shells towed through the air some 2 kilometres away. MBDA's tests have also helped to debunk the science-fiction idea that reflective armour would defend against laser weapons. They found that any dust on the mirrored surface would get burned in, and lead to the destruction of the target even faster than with a non-reflective surface.

Markus Martinstetter from MBDA's Future Systems Directorate argues that high-precision targeting minimizes the chances of accidentally hurting bystanders while trying to shoot down targets, especially compared with conventional explosives. "There is no risk from fragmenting ammunition and we only start the irradiation when the aim point is exactly on target," he says.

Lockheed Martin is also working on laser weapons that can take on targets that are more complex or farther away than can be tackled by its low-cost ADAM system. In March, for example, the company reported that its Advanced Test High Energy Asset (ATHENA) system could disable the running engine of a small truck mounted on a test platform. ATHENA uses a similar adaptive-optics system to the Airborne Laser, coupled with Lockheed's Accelerated Laser Demonstration Initiative (ALADIN) fibre-laser system.

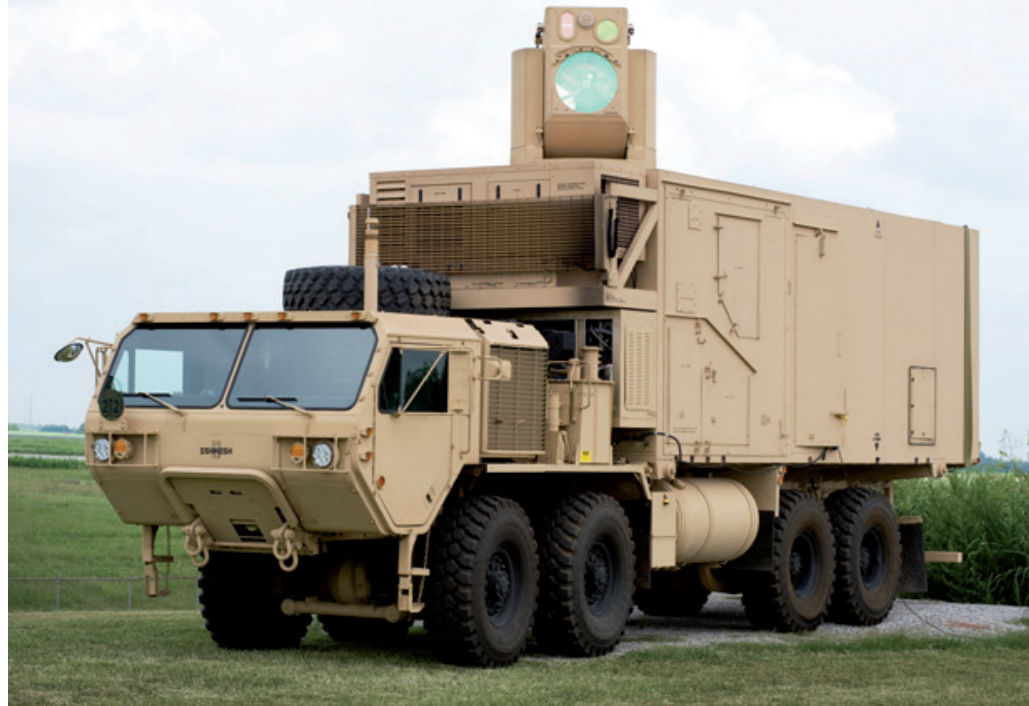

The High Energy Laser Mobile Demonstrator can take down drones.

ALADIN combines the output of several fibre lasers, each with a slightly different wavelength, into a single $30-\mathrm{kW}$ beam. This 'wavelength beam combining' approach originated at the Lincoln Laboratory ${ }^{5}$ and is similar to methods that channel Internet traffic into fibre-optic cables. Fan notes that this method is easier than coherent combining, but gives better-quality beams than incoherent combining, so it can more easily hit smaller targets from longer distances.

Jason Ellis, a visiting fellow at the CNAS and lead author of the think tank's laser-weapons report ${ }^{1}$, says that such developments convince him that fibre-laser weapons are coming of age - and that emerging advances could take them to hundreds of kilowatts and extend their range to hundreds of kilometres.

Despite such advances, a February 2014 poll $^{6}$ of US national-security specialists found that just one-fifth believed that directed-energy weapon technologies would be mature within a decade.

Michael Carter, a programme manager for photon science at Lawrence Livermore National Laboratory in Livermore, California, cautions that today's lasers are a very long way from their science-fiction counterparts. "They're not yet the Star Trek phaser," he says. "People talk about speed-of-light engagement, but it still takes time to demolish targets. At the most basic level, if you can't see it - if there's too much rain or fog - your laser can't hit it." He suggests that the greatest value of the current generation of demonstration systems may be in working out how to handle such broader challenges before better lasers emerge. "Don't mistake what they're doing on the USS Ponce for a new strategic superiority," Carter warns. "It may be the first step in that direction but it's not going to change the game by itself."

Even the weapons companies are cautious not to overstate their case. For example, MBDA expects that it will take 3-5 years for truly operational systems to appear even in the tens of kilowatts range. And in some circumstances - such as a foggy day - conventional weapons will always be more effective. "You give the defender of the future both, and put the choice in their hands," DeYoung recommends.

Despite their modest capabilities, Scharre claims that fibre-laser weapons could find a niche in US military defence in 5-10 years. "They may not be as grand and strategic as the Star Wars concept," he says, "but they could save lives, protect US bases, ships and service members."

Andy Extance is a freelance writer in Exeter, $U K$.

1. Ellis, J. D. Directed-Energy Weapons: Promise and Prospects (CNAS, 2015); available at http://go.nature.com/eipivo

2. Sprangle, P., Peñano, J. \& Hafzi, B. J. Directed Energy 2, 71-95 (2006).

3. Sprangle, P., Ting, A., Penano, J., Fischer, R. \& Hafizi, B. IEEE J. Quantum Elect. 45, 138-148 (2009).

4. Mohring, B. et al. Proc. SPIE 8733, 873304 (2013).

5. Daneu, V. et al. Opt. Lett. 25, 405-407 (2000).

6. FitzGerald, B. \& Sayler, K. Creative Disruption: Technology, Strategy and the Future of the Global Defense Industry (CNAS, 2014); available at http://go.nature. com/7iwlaq 